\title{
Detecting Exoplanets with the Xallarap Microlensing Effect
}

\author{
Sohrab Rahvar* \\ Department of Physics, Sharif University of Technology, P.O. Box 11365-9161, Tehran, Iran \\ E-mail: rahvar@sharif.edu

\section{Martin Dominik ${ }^{\dagger}$} \\ SUPA, University of St Andrews, School of Physics \& Astronomy, North Haugh, St Andrews, \\ KY16 9SS, United Kingdom \\ md35est-andrews.ac.uk
}

\begin{abstract}
One of the successful techniques for the detection of exoplanets relies on the magnification of an observed star resulting from microlensing by a foreground star orbited by a planetary companion. We propose an alternative method for the detection of exoplanets by microlensing, in which the orbital motion of a planet around the source star induces a small motion of the star around the common barycentre, which leads to detectable deviations from the ordinary symmetric microlensing light curve. We show that favourable events for such deviations to occur involve lenses close to the source star and Einstein-radius crossing times substantially larger than the planet's orbital period. From a Monte-Carlo simulation, we find that a monitoring programme of Galactic bulge stars, capable of providing a 2-hour sampling and $2 \%$ photometric accuracy, can detect planets of Jupiter mass with a detection efficiency of around $1 \%$.
\end{abstract}

The Manchester Microlensing Conference: The 12th International Conference and ANGLES Microlensing Workshop

January 21-25 2008

Manchester, UK

\footnotetext{
${ }^{*}$ Speaker.

${ }^{\dagger}$ Royal Society University Research Fellow
} 


\section{Introduction}

Gravitational microlensing, i.e. the transient brightening of an observed star due to the bending of light caused by the gravitational field of an intervening foreground star, was considered by Einstein as early as 1912 [1], but he concluded that "there is no great chance of observing this phenomenon" [2]. Only several decades of advance in technology enabled the first reported discovery of a microlensing event [3], following the suggestion by Paczyński to use the technique as a tool for detecting compact matter in the Galactic halo [4].

After one decade of monitoring of Large and Small Magellanic Clouds for searching MACHOs in the Galactic halo, the conclusion is that "there is almost no MACHO in the halo" [5]. While the mission of gravitational microlensing in searching MACHOs in the Galactic halo is almost finished, however this technique can be used as an astrophysical tool for stellar-physics studies and the detection of low-mass planets. On top of the ordinary brightening of the observed source star during a microlensing event, a planetary companion to the lens star can cause a further short blip or dip. A super-Jupiter was the first planet detected by this technique [6], but its sensitivity even reaches below the mass of Earth, even for ground-based observations [7, 8].

Here, we discuss another channel for revealing the existence of extra-solar planets from the study of microlensing light curves [9]. While the standard approach leads to the detection of planets orbiting the lens star, we study the effect of a the planet accompanying the source star. Given that both the planet and its host star orbit the common barycentre, there is a small motion of the source star, which periodically alters the line-of-sight and thereby the relative lens-source position, so that the orbiting planet finally reveals its presence by means of an observed change in magnification. This effect is in close analogy to the parallax effect caused by the orbital motion of the Earth with just exchanging the roles of source and observer as well as of planet and host star. Observation of the annual parallax in a microlensing event was first reported by the MACHO group [10], whereas effects of source binarity have been addressed by various authors [11-14]. Due to its geometric reflection of the parallax effect, this is sometimes called the "xallarap" effect. The observational distinction between these is frequently not that easy. For instance, a systematic analysis of 22 microlensing parallax candidate events [15] explicitly found $23 \%$ of them being strongly affected by 'xallarap'.

We lay out the theoretical basis of the effect of planets orbiting the source star on its microlensing light curve in Sect. 2, present the results of a Monte-Carlo simulation for estimating the number of planets that can be expected to be detected through this channel in Sect. 3, and close with final conclusions in Sect. 4.

\section{Orbiting planets and their effect on the microlensing light curve}

\subsection{Ordinary microlensing light curves}

According to the theory of General Relativity, light passing near a star of mass $M$ at an impact parameter $b$ is bent by the angle $\alpha=(4 G M) /\left(b c^{2}\right)$, where $G$ is the universal gravitational constant and $c$ is the speed of light. For a point-like deflector, there are two possible light rays from the source to the observer, leading to two images for any point source, except for the case of perfect 
alignment between observer, lens, and source star, where a circle with the angular 'Einstein' radius

$$
\theta_{\mathrm{E}}=\sqrt{\frac{4 G M}{c^{2}}\left(D_{\mathrm{L}}^{-1}-D_{\mathrm{S}}^{-1}\right)}
$$

forms, where $D_{\mathrm{L}}$ and $D_{\mathrm{S}}$ are the distances of the lens and source from the observer, respectively. For typical distances within the Milky Way, the size of the angular Einstein radius implies angular separations between the images of the order of 1 milli-arcsecond, so that these cannot be resolved by current ground-based telescopes. However, the change in solid angle subtained on the sky by the images as compared to the source star corresponds to the source star appearing brighter by the magnification factor

$$
A(u)=\frac{u^{2}+2}{u \sqrt{u^{2}+4}}
$$

where $u=|\vec{u}|$ is the angular separation between lens and source in units of the angular Einstein radius. Given that the relative positions between source, lens, and observer change with time, we can detect a variation in brightness of the source star during a so-called gravitational microlensing event exhibiting a characteristic light curve.

For 'ordinary' microlensing events, both the source and lens star are considered to be point-like objects, and their relative proper motion of is uniform, $\vec{\mu}=\vec{\mu}_{\mathrm{S}}-\vec{\mu}_{\mathrm{L}}=\mu(\cos \psi, \sin \psi)$. Normalizing the relative angular separation between the lens and source to $\theta_{\mathrm{E}}$,

$$
u=\sqrt{u_{0}^{2}+\omega_{\mathrm{E}}^{2}\left(t-t_{0}\right)^{2}}
$$

where $\omega_{\mathrm{E}}=\mu / \theta_{\mathrm{E}}$. In the literature, rather than $\omega_{\mathrm{E}}$, a time-scale $t_{\mathrm{E}}=\omega_{\mathrm{E}}^{-1}$ is used more frequently. The magnification as function of time is therefore completely described by the parameters $u_{0}, t_{\mathrm{E}}$, and $t_{0}$, which can be determined from a fit to the measured light curve.

\subsection{Parallax effect}

If the Earth's acceleration is not negligible, the apparent trajectory of the deflector with respect to the line-of-sight becomes a cycloid rather than a straight line (assuming the orbit being nearly circular). The magnification of the source star thereby becomes affected by the revolution of the Earth around the Sun, providing an annual parallax. Naturally, this effect becomes more prominent for microlensing events of longer duration, i.e. several months or more ([10]).

The relative parallax of lens and source normalized to $\theta_{\mathrm{E}}$, namely

$$
\pi_{\mathrm{E}}=\frac{1 \mathrm{AU}}{\theta_{\mathrm{E}}}\left(\frac{1}{D_{\mathrm{L}}}-\frac{1}{D_{\mathrm{S}}}\right)
$$

provides the relevant scale of this effect. Using this definition, the orbital motion of the Earth leads to a further effective relative motion between lens and source star as seen from Earth that reads

$$
\begin{aligned}
& \hat{u}_{1}(t)=-\pi_{\mathrm{E}} \sin \beta \cos \zeta(t), \\
& \hat{u}_{2}(t)=\pi_{\mathrm{E}} \sin \zeta(t),
\end{aligned}
$$

where $\zeta(t)=[(2 \pi) / P] t-\varphi$ with $P$ denoting the orbital period and $\varphi$ the orbital phase, while $\beta$ is the inclination of the Earth's orbit with respect to the line-of-sight, which coincides with the 
ecliptical latitude of the observed source star. Combining this motion with the uniform motion of the lens with respect to the source, while adopting the orientation angle $\psi$ of the source trajectory as referring to the $\left(\hat{u}_{1}, \hat{u}_{2}\right)$ coordinate axes, we find for the absolute value of the total relative positional shift $\vec{u}(t)=\vec{u}_{L S}(t)+\hat{\vec{u}}(t)$

$$
\begin{aligned}
& {[u(t)]^{2}=u_{0}^{2}+\omega_{\mathrm{E}}^{2}\left(t-t_{0}\right)^{2}+2 \pi_{\mathrm{E}}\left\{\sin \zeta(t)\left[u_{0} \cos \psi+\omega_{\mathrm{E}}\left(t-t_{0}\right) \sin \psi\right]+\right.} \\
& \left.\quad+\sin \beta \cos \zeta(t)\left[u_{0} \sin \psi-\omega_{\mathrm{E}}\left(t-t_{0}\right) \cos \psi\right]\right\}+ \\
& +\pi_{\mathrm{E}}^{2}\left[\sin ^{2} \zeta(t)+\sin ^{2} \beta \cos ^{2} \zeta(t)\right] .
\end{aligned}
$$

\subsection{Xallarap effect}

Now imagine the source star and a surrounding planet orbiting their common barycentre. As compared to the annual parallax due to the revolution of the Earth around the Sun, one faces a situation with regard to microlensing that just corresponds to the inversion of the direction of light rays. It is therefore obvious that both effects of periodic displacement are to take exactly the same form.

In analogy to the discussion presented in the previous subsection, let us therefore define a parameter

$$
\chi_{\mathrm{E}}=\frac{m_{\mathrm{p}}}{m} \frac{a}{D_{\mathrm{S}} \theta_{\mathrm{E}}}>0
$$

where $a$ is the semi-major axis of the planetary orbit. If, for simplicity, we consider a circular orbit, the orbital motion of the planet causes the angular position of the source normalized to the Einstein angle change with time as

$$
\begin{aligned}
& \hat{u}_{1}(t)=\chi_{\mathrm{E}} \sin i \cos \zeta(t), \\
& \hat{u}_{2}(t)=-\chi_{\mathrm{E}} \sin \zeta(t),
\end{aligned}
$$

where $\zeta(t)=\Omega\left(t-t_{0}\right)-\varphi$, with $\Omega=(2 \pi) / P$, and $i$ denoting the orbital inclination. The effective relative position between source and lens is then given by

$$
\begin{aligned}
& {[u(t)]^{2}=u_{0}^{2}+\omega_{\mathrm{E}}^{2}\left(t-t_{0}\right)^{2}-2 \chi_{\mathrm{E}}\left\{\sin \zeta(t)\left[u_{0} \cos \psi+\omega_{\mathrm{E}}\left(t-t_{0}\right) \sin \psi\right]+\right.} \\
& \left.\quad+\sin i \cos \zeta(t)\left[u_{0} \sin \psi-\omega_{\mathrm{E}}\left(t-t_{0}\right) \cos \psi\right]\right\}+ \\
& +\chi_{\mathrm{E}}^{2}\left[\sin ^{2} \zeta(t)+\sin ^{2} \beta \cos ^{2} \zeta(t)\right] .
\end{aligned}
$$

One indeed realizes that Eqs. (2.6) and (2.9) have an identical form, where only $\pi_{\mathrm{E}} \leftrightarrow-\chi_{\mathrm{E}}$ and $\beta \leftrightarrow i$. The additional sign arises from the fact that here we are considering the motion of the observable star rather than the unobservable planet, as we do when observing from Earth. Given that $u_{0}$ refers to the minimal impact with respect to the barycentre, the epoch $t_{0}$ does in general not mark a maximum of the magnification.

\section{Planet detection}

\subsection{Strength of exoplanet signal}

In order to obtain an estimate of the strength of the signal, and to figure out the favoured scenarios, let us study the components of the relevant parameter $\chi_{\mathrm{E}}=\left(m_{\mathrm{p}} / M\right)\left[a /\left(D_{\mathrm{S}} \theta_{\mathrm{E}}\right)\right]$ a bit 
further. At the source distance $D_{\mathrm{S}}$, the angular Einstein radius $\theta_{\mathrm{E}}$ corresponds to a physical size

$$
\begin{aligned}
D_{\mathrm{S}} \theta_{\mathrm{E}} & =\sqrt{\frac{4 G M}{c^{2}}\left(D_{\mathrm{S}}-D_{\mathrm{L}}\right) \frac{D_{\mathrm{S}}}{D_{\mathrm{L}}}} \\
& =2.8 \mathrm{AU}\left(\frac{M}{1 M_{\odot}}\right)^{1 / 2}\left(\frac{D_{\mathrm{S}}-D_{\mathrm{L}}}{1 \mathrm{kpc}}\right)^{1 / 2}\left(\frac{D_{\mathrm{L}}}{D_{\mathrm{S}}}\right)^{-1 / 2}
\end{aligned}
$$

Given that Kepler's third law relates the orbital period $P$ to the major semi-axis $a$ as

$$
P=2 \pi \sqrt{\frac{a^{3}}{G\left(m_{\star}+m_{\mathrm{p}}\right)}}
$$

where $m_{\star}$ is the mass of source star and $m_{\mathrm{p}}$ is the mass of its planet, we can insert (3.1) and (3.2) into Eq. (2.7) to obtain $\chi_{\mathrm{E}}$ in terms of fundamental properties of the planet, the source star, and the lens star as

$$
\begin{aligned}
& \chi_{\mathrm{E}}=6.4 \times 10^{-4} \frac{m_{\mathrm{p}}}{M_{\mathrm{jup}}}\left(\frac{m}{1 M_{\odot}}\right)^{-2 / 3}\left(\frac{M}{0.3 M_{\odot}}\right)^{-1 / 2} \times \\
& \times\left(\frac{P}{1 \mathrm{yr}}\right)^{2 / 3}\left(\frac{D_{\mathrm{S}}-D_{\mathrm{L}}}{1 \mathrm{kpc}}\right)^{-1 / 2}\left(\frac{D_{\mathrm{L}}}{D_{\mathrm{S}}}\right)^{1 / 2} .
\end{aligned}
$$

Thereby, we see that large orbital periods $P$ and small lens-source distances $D_{\mathrm{S}}-D_{\mathrm{L}}$ are preferred. Another relevant parameter is the event time-scale $t_{\mathrm{E}}=\theta_{\mathrm{E}} / \mu$, which reads

$$
t_{\mathrm{E}}=17 \mathrm{~d}\left(\frac{M}{0.3 M_{\odot}}\right)^{-1 / 2}\left(\frac{D_{\mathrm{S}}-D_{\mathrm{L}}}{1 \mathrm{kpc}}\right)^{1 / 2}\left(\frac{D_{\mathrm{L}}}{D_{\mathrm{S}}}\right)^{1 / 2} \times\left(\frac{D_{\mathrm{L}} \mu}{160 \mathrm{~km} \mathrm{~s}^{-1}}\right)^{-1} .
$$

For the Galactic bulge-bulge lensing, $D_{\mathrm{L}} \mu \sim 160 \mathrm{~km} \mathrm{~s}^{-1}$ and $D_{\mathrm{S}}-D_{\mathrm{L}} \sim 1 \mathrm{kpc}$, which provides a 'typical' value of $t_{\mathrm{E}} \sim 17 \mathrm{~d}$. Despite the fact that $\chi_{\mathrm{E}}$ increases with $P$, the lack of characteristic features while the source is magnified makes deviations quite small for $P>t_{\mathrm{E}}$, so that the sample light curve shown in Fig. 1 consequently refers to a parameter set with $P<t_{\mathrm{E}}$. This shows that events with large $t_{\mathrm{E}}$ provide larger prospects for planet detection through our proposed channel.

\subsection{Estimating planet detectability from a Monte-Carlo simulation}

After having identified the basic scenarios, we carried out a Monte-Carlo simulation in order to study the detectability of planets as a function of various parameters that describe the lens star, the source star, and its orbiting planet. For simplicity, we have assumed circular orbits $(\varepsilon=0)$, so that the source magnification $A[u(t)]$ is described by the 8 parameters $\left(u_{0}, t_{0}, \omega_{\mathrm{E}}, \chi_{\mathrm{E}}, \psi, \Omega, i, \varphi\right)$.

Without loss of generality, we set $t_{0}=0$. Moreover, we consider planets of mass $m_{\mathrm{p}}=1 M_{\text {jup }}$ orbiting a source star of $m_{\star}=1 M_{\odot}$, while adopting a Galactic bulge mass function [16] for the lens stars. With these choices, according to Eq. (3.3), the strength parameter $\chi_{\mathrm{E}}$ then becomes a function of the lens and source distances $D_{\mathrm{L}}$ and $D_{\mathrm{S}}$, and the orbital frequency $\Omega=(2 \pi) / P$, where $P$ denotes the orbital period. We choose the location of lens and source stars according to the Galactic bulge mass density, and draw the orbital period $P$ from a uniform distribution in $\lg [P /(1 \mathrm{~d})]$ ranging between $3 \mathrm{~d}$ and $1 \mathrm{yr}$. While we adopt a 'natural' uniform distribution of impact parameters $u_{0} \in[0,1]$, not taking into account any selection bias by the experiment, the 

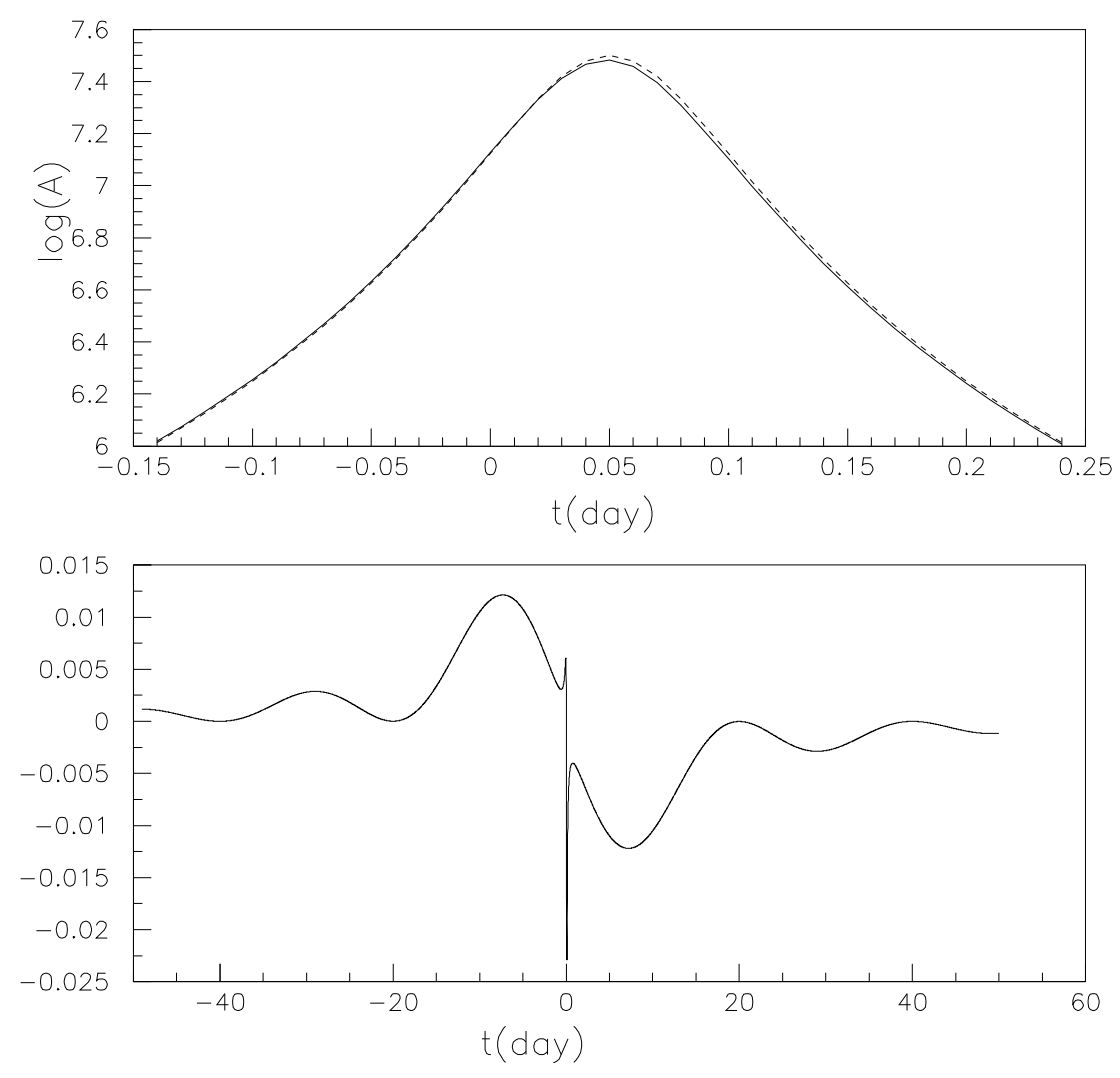

Figure 1: A sample light curve affected by the motion of the source star around its barycentre with an orbiting planet. For the underlying parameters, we assumed $\chi_{\mathrm{E}}=10^{-3}, P=20 \mathrm{~d}, t_{\mathrm{E}}=50 \mathrm{~d}, u_{0}=10^{-3}$, $t_{0}=0, \varphi=30^{\circ}$ and $i=80^{\circ}$. In the upper panel, the solid line represents the xallarap light curve, whereas the dashed line is the best fit to an ordinary microlensing light curve. The lower panel shows the difference between the two curves. Potential finite-source effects that can particularly modify the peak of the light curve have been ignored.

phase angle $\varphi$, orientation angle $\psi$, and inclination angle $i$ are all naturally uniformly distributed, where $\varphi, \psi \in[0,2 \pi)$ and $i \in[0, \pi / 2]$.

Rather than confusing ourselves and others with too many details, we take a simple pragmatic approach in adopting a photometric accuracy of $2 \%$ for all obervations and a sampling interval of 2 hours, without any loss due to bad weather or other causes. A quantitative measure for the detectability of a planetary signal then results from a likelihood-ratio test, involving the respective $\chi^{2}$ minima for best-fitting models with or without a planet, namely $\left(\chi_{\min }^{2}\right)^{(0)}$ and $\left(\chi_{\min }^{2}\right)^{\text {planet }}$. In fact, with $\Delta \chi^{2} \equiv\left(\chi_{\min }^{2}\right)^{(0)}-\left(\chi_{\min }^{2}\right)^{\text {planet }}$ following a $\chi^{2}$-distribution with 5 degrees of freedom, one finds a probability $P\left(\Delta \chi^{2} \geq 11.07\right)=0.05$ for such a difference to arise. At this significance level, we therefore reject the hypothesis that an ordinary light curve explains the data provided that $\Delta \chi^{2} \geq 11.07$, and claim the detection of a planetary signal. Figure 2 shows the detectability 

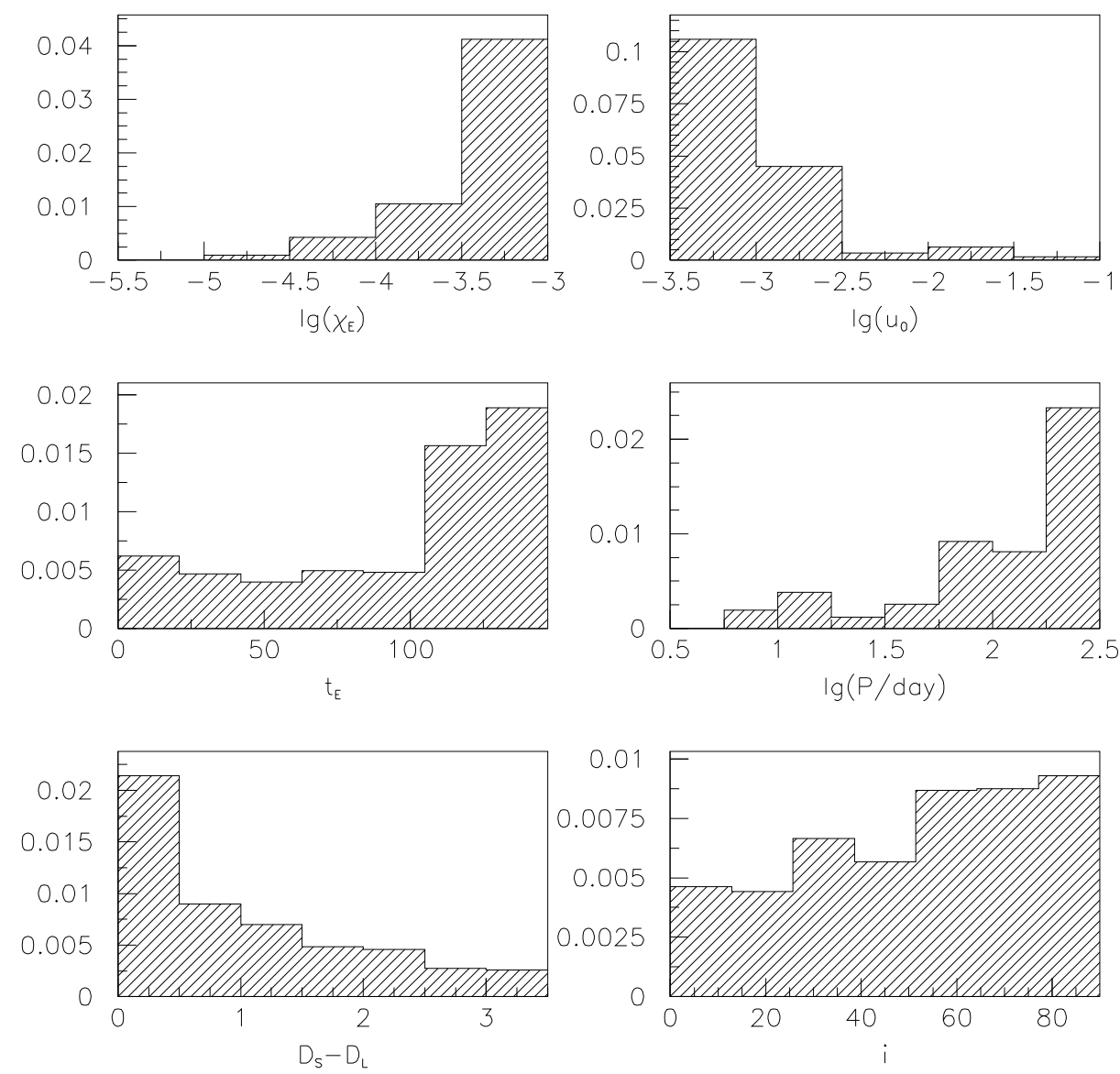

Figure 2: Detection efficiency for a planet of mass $m_{\mathrm{p}}=1 M_{\text {jup }}$ orbiting a Galactic bulge star as a function of $\chi_{\mathrm{E}}, u_{0}, t_{\mathrm{E}}, P, D_{\mathrm{S}}-D_{\mathrm{L}}$ and $i$. The overall observational efficiency is in the order of $1 \%$.

according to this criterion as a function of various parameters.

\section{Conclusion}

We show that an alternative channel for the detection of extra-solar planets by microlensing is provided by measuring the alteration of the source magnification resulting from its orbital motion around the common barycentre with an unseen planetary companion, as opposed to the standard channel where a planet orbiting the lens stars alters the bending angle and thereby the observed magnification. We derived a formalism for the deviation of light curves in analogy to the parallax effect resulting from the revolution of the Earth around the Sun. While the annual parallax is more prominent for lens stars close to the observer, the detecting of planets orbiting the source stars by means of their motion around the barycentre is best supported by lens stars close to the source star. 
From a Monte-Carlo simulation, we find that on events towards the Galactic bulge sampled every 2 hours with $2 \%$ photometric accuracy, the probability for detecting a Jupiter-mass planet is around $1 \%$. Therefore, not only can future observations possibly provide detections through this channel, but such might already lie hidden in the data that have already been acquired.

\section{References}

[1] J. Renn, T. Sauer, J. Stachel, The origin of gravitational lensing: a postscript to Einstein's 1936 Science paper, Science, 275 (1997), 184.

[2] A. Einstein, Lens-Like Action of a Star by the Deviation of Light in the Gravitational Field, Science, 84 (1936), 506

[3] C. Alcock et al.,Possible Gravitational Microlensing of a Star in the Large Magellanic Cloud, Nature, 365 (1993) 621.

[4] B. Paczyński,Gravitational microlensing by the galactic halo, ApJ, 304 (1986), 1

[5] P. Tisserand, et al,Limits on the Macho content of the Galactic Halo from the EROS-2 Survey of the Magellanic Clouds, A\&A, 467 (2007), 387

[6] I. A. Bond et al.,OGLE 2003-BLG-235/MOA 2003-BLG-53: A Planetary Microlensing Event, ApJ, 606 (2004), L155

[7] D. P. Bennett, S. H. Rhie,Detecting Earth-Mass Planets with Gravitational Microlensing, ApJ, 472 (1996), 660

[8] M. Dominik et al.,An anomaly detector with immediate feedback to hunt for planets of Earth mass and below by microlensing, MNRAS,380 (2007), 792

[9] S. Rahvar, M. Dominik, Planetary microlensing signals from the orbital motion of the source star around the common barycentre,(2008, MNRAS submitted).

[10] C. Alcock et al.,First Observation of Parallax in a Gravitational Microlensing Event, ApJ, 454 (1995), L125

[11] K. Griest, W. Hu, Effect of binary sources on the search for massive astrophysical compact halo objects via microlensing, ApJ, 397 (1992), 362

[12] C. Han, A. Gould, Einstein Radii from Binary-Source Lensing Events, ApJ, 480 (1997), 196

[13] B. Paczyński, Binary Source Parallactic Effect in Gravitational Micro-lensing, preprint astro-ph/9711007

[14] M. Dominik,Galactic microlensing with rotating binaries, A\&A 329 (1998), 361.

[15] S. Poindexter et. al., Systematic Analysis of 22 Microlensing Parallax Candidates, ApJ, 633 (2005), 914.

[16] G. Chabrier, The Galactic Disk Mass Function: Reconciliation of the Hubble Space Telescope and Nearby Determinations, ApJ, 586 (2003), L133. 